\title{
AUSLANDER GENERATORS AND HOMOLOGICAL CONJECTURES
}

\author{
JIAQUN WEI \\ Institute of Mathematics, School of Mathematical Sciences, \\ Nanjing Normal University, Nanjing 210023, P. R. China \\ e-mail:weijiaqun@njnu.edu.cn
}

(Received 19 December 2012; revised 5 April 2013; accepted 9 April 2013; first published online 30 August 2013)

\begin{abstract}
Let $A$ be an artin algebra with representation dimension not more than 3. Assuming that ${ }_{A} V$ is an Auslander generator and $M \in \operatorname{add}_{A} V$, we show that both findim $\left(\operatorname{End}_{A} M\right)$ and findim $\left(\operatorname{End}_{A} M\right)^{o p}$ are finite, and consequently the Gorenstein symmetry conjecture, the Wakamatsu-tilting conjecture and the generalized Nakayama conjecture hold for $\operatorname{End}_{A} M$.
\end{abstract}

2010 Mathematics Subject Classification. 16E05, 16E10, 16G10.

1. Introduction and main result. Let $A$ be an artin algebra. The finitistic dimension of $A$, denoted by findim $A$, is defined to be the supremum of the projective dimensions of all finitely generated modules of finite projective dimension. The famous finitistic dimension conjecture asserts that findim $A$ is always finite.

Igusa and Todorov [3] presented a good way to test the finitistic dimension conjecture. In particular, they proved that $\operatorname{fin} \operatorname{dim} A$ is finite, provided that the representation dimension of $A$, denoted by $\operatorname{repdim} A$, is not more than 3 . Recall that $\operatorname{repdim} A=\inf \left\{\operatorname{gd}\left(\operatorname{End}_{A} V\right) \mid V\right.$ is a generator-cogenerator $\}$, where gd denotes the global dimension and $\operatorname{End}_{A} V$ denotes the endomorphism algebra of ${ }_{A} V$. A generatorcogenerator such as $\operatorname{repdim} A=\operatorname{gd}\left(\operatorname{End}_{A} V\right)$ is called an Auslander generator. In general, an artin algebra may have many Auslander generators, see for instance [2].

Our main result is stated as follows.

TheOrem 1.1. Let $A$ be an artin algebra with $\operatorname{repdim} A \leq 3$. Assume that ${ }_{A} V$ is an Auslander generator. Then both findim $\left(\operatorname{End}_{A} M\right)$ and $\operatorname{findim}\left(\operatorname{End}_{A} M\right)^{\text {op }}$ are finite, whenever $M \in \operatorname{add}_{A} V$.

Theorem 1.1 generalizes the main result of [6]. It is not known if findim $A^{o p}$ is finite, provided that findim $A$ is finite in general, where $A^{o p}$ denotes the opposite algebra of $A$.

We recall the following well-known conjectures (see, for instance, $[\mathbf{1}, \mathbf{4}]$ ). Here $E$ is an artin algebra.

Gorenstein symmetry conjecture. $\operatorname{id}\left({ }_{E} E\right)<\infty$ if and only if $\operatorname{id}\left(E_{E}\right)<\infty$, where id denotes the injective dimension.

Wakamatsu-tilting conjecture. Let ${ }_{E} \omega$ be a Wakamatsu-tilting module.

(1) If $\operatorname{pd}_{E} \omega<\infty$, then $\omega$ is tilting.

(2) If $\operatorname{id}_{E} \omega<\infty$, then $\omega$ is co-tilting. 
Generalized Nakayama conjecture. Each indecomposable injective $E$-module occurs as a direct summand in the minimal injective resolution of ${ }_{E} E$.

It is well known that the finitistic dimension conjecture hold for $E$ and $E^{o p}$ implies that all the above conjectures hold. Hence, we have the following corollary.

Corollary 1.2. Let $A$ be an artin algebra with $\operatorname{repdim} A \leq 3$. Assume that ${ }_{A} V$ is an Auslander generator. Then the Gorenstein symmetry conjecture, the Wakamatsutilting conjecture and the generalized Nakayama conjecture hold for $\operatorname{End}_{A} M$ whenever $M \in \operatorname{add}_{A} V$.

Since representation-finite algebras and torsionless-finite algebras have representation dimension of not more than 3 (see [5]), we obtain the following result as special cases.

COROLlary 1.3. Let $A=\operatorname{End}_{\Lambda} M$, where $\Lambda$ and $M$ satisfy one of the following conditions:

(1) $\Lambda$ is a representation-finite algebra and $M$ is any $\Lambda$-module, or

(2) $\Lambda$ is a torsionless-finite algebra and $M$ is torsionless or co-torsionless or a direct sum of torsionless and co-torsionless modules.

Then both $\operatorname{fin} \operatorname{dim} A$ and $\operatorname{fin} \operatorname{dim} A^{\text {op }}$ are finite. In particular, the Gorenstein symmetry conjecture, the Wakamatsu-tilting conjecture and the generalized Nakayama conjecture hold for $A$.

\section{The proof.}

Let $A$ be an artin algebra. We denote $A-\bmod$ the category of all finite generated left $A$-modules. Assume $M \in A-\bmod$. We denote $\operatorname{pd}_{A} M$ the projective dimension of ${ }_{A} M$ and $\Omega_{A}^{i} M$ the $i$ th syzygy of $M$. Throughout the paper, $\mathbf{D}$ denotes the usual duality functor between $A-\bmod$ and $A^{o p}$-mod.

The following lemma is well known.

LEMMA 2.1. Let $A$ be an artin algebra and let $V$ be a generator-cogenerator in $A$-mod. The following are equivalent for a non-negative integer $n$.

(1) $\operatorname{gd}\left(\operatorname{End}_{A} V\right) \leq n+2$.

(2) For any $X \in A$-mod, there is an exact sequence $0 \rightarrow V_{n} \rightarrow \cdots \rightarrow V_{1} \rightarrow$ $V_{0} \rightarrow X \rightarrow 0$ with each $V_{i} \in \operatorname{add}_{A} V$ such that the corresponding sequence induced by the functor $\operatorname{Hom}_{A}(V,-)$ is also exact.

The following lemma collects some important properties of the Igusa-Todorov functor introduced in [3].

LemMA 2.2. For any artin algebra $A$, there is a functor $\Psi$ which is defined on the objects of $A-\bmod$ and takes non-negative integers as values, such that

(1) $\Psi(M)=\operatorname{pd}_{A} M$, provided that $\mathrm{pd}_{A} M<\infty$.

(2) $\Psi(X) \leq \Psi(Y)$ whenever $\operatorname{add}_{A} X \subseteq \operatorname{add}_{A} Y$. The equation holds in case $\operatorname{add}_{A} X=\operatorname{add}_{A} Y$.

(3) If $0 \rightarrow X \rightarrow Y \rightarrow Z \rightarrow 0$ is an exact sequence in $A-\bmod$ with $\operatorname{pd}_{A} Z<\infty$, then $\operatorname{pd}_{A} Z \leq \Psi(X \oplus Y)+1$.

Let $A$ be an artin algebra and $M \in A-\bmod$ with $E=\operatorname{End}_{A} M$. Then $M$ is also a right $E$-module. It is well known that $\left(M \otimes_{E}-, \operatorname{Hom}_{A}(M,-)\right)$ is a pair of adjoint functors and that, for any $E$-module $Y$, there is a canonical homomorphism $\sigma_{Y}$ : 
$Y \rightarrow \operatorname{Hom}_{A}\left(M, M \otimes_{E} Y\right)$ defined by $n \rightarrow[t \rightarrow t \otimes n]$. It is easy to see that $\sigma_{Y}$ is an isomorphism, provided that $Y$ is a projective $E$-module.

The following lemma is essential.

Lemma 2.3. Let $M \in A-\bmod$ and $E=\operatorname{End}_{A} M$. Then, for any $X \in E-\bmod$, $\Omega_{E}^{2} X \simeq \operatorname{Hom}_{A}(M, Y)$ for some $Y \in A-\bmod$.

Proof. Consider the exact sequence

$$
0 \rightarrow \Omega_{E}^{2} X \rightarrow E_{1} \rightarrow E_{0} \rightarrow X \rightarrow 0
$$

with $E_{0}, E_{1} \in E-$ mod projective. Applying the functor $M \otimes_{E}-$, we obtain an induced exact sequence

$$
0 \rightarrow Y \rightarrow M \otimes_{E} E_{1} \rightarrow M \otimes_{E} E_{0} \rightarrow M \otimes_{E} X \rightarrow 0,
$$

for some $Y \in A-\bmod$. Now applying the functor $\operatorname{Hom}_{A}(M,-)$, we further have an induced exact sequence

$$
0 \rightarrow \operatorname{Hom}_{A}(M, Y) \rightarrow \operatorname{Hom}_{A}\left(M, M \otimes_{E} E_{1}\right) \rightarrow \operatorname{Hom}_{A}\left(M, M \otimes_{E} E_{0}\right) .
$$

Moreover, there is the following commutative diagram:

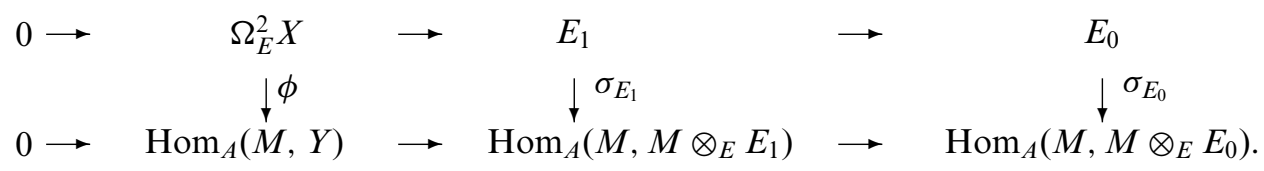

Since $E=\operatorname{End}_{A} M$ and $E_{0}, E_{1} \in \operatorname{add}_{E} E$, the canonical homomorphisms $\sigma_{E_{0}}$ and $\sigma_{E_{1}}$ are isomorphisms. It follows that $\Omega_{E}^{2} X \simeq \operatorname{Hom}_{A}(M, Y)$.

Proof of Theorem 1.1. Let $E:=\operatorname{End}_{A} M$. Suppose that $X \in E-\bmod$ and $\operatorname{pd}_{E} X<$ $\infty$. Then $\operatorname{pd}_{E}\left(\Omega_{E}^{2} X\right)<\infty$. Moreover, $\Omega_{E}^{2} X \simeq \operatorname{Hom}_{A}(M, Y)$ for some $Y \in A-$ mod, by Lemma 2.3. Since ${ }_{A} V$ is a generator-cogenerator such that $\operatorname{gd}\left(\operatorname{End}_{A} V\right) \leq 3$, by Lemma 2.1 we obtain an exact sequence

$$
0 \rightarrow V_{1} \rightarrow V_{0} \rightarrow Y \rightarrow 0(\dagger)
$$

with $V_{0}, V_{1} \in \operatorname{add}_{A} V$ such that the corresponding sequence induced by the functor $\operatorname{Hom}_{A}(V,-)$ is also exact. Note that $M \in \operatorname{add}_{A} V$, so the sequence ( $\left.\dagger\right)$ also stays exact under the functor $\operatorname{Hom}_{A}(M,-)$. Thus, we have the following exact sequence in $E-\bmod$ :

$$
0 \rightarrow \operatorname{Hom}_{A}\left(M, V_{1}\right) \rightarrow \operatorname{Hom}_{A}\left(M, V_{0}\right) \rightarrow \operatorname{Hom}_{A}(M, Y) \rightarrow 0 .
$$

Now by Lemma 2.2, we have that

$$
\begin{aligned}
\operatorname{pd}_{E} X & \leq \operatorname{pd}_{E}\left(\Omega_{E}^{2} X\right)+2 \\
& =\operatorname{pd}_{E}\left(\operatorname{Hom}_{A}(M, Y)\right)+2 \\
& \leq \Psi\left(\operatorname{Hom}_{A}\left(M, V_{0}\right) \oplus \operatorname{Hom}_{A}\left(M, V_{1}\right)\right)+1+2 \\
& \leq \Psi\left(\operatorname{Hom}_{A}(M, V)\right)+1+2<\infty .
\end{aligned}
$$


It follows that findim $E$ is finite.

Now consider algebras $A^{o p}$ and $\left.E^{o p}\left(=\operatorname{End}_{A} M\right)^{o p}\right)$. Since ${ }_{A} V$ is a generatorcogenrerator in $A-\bmod , A^{o p} \mathbf{D} V$ is also a generator-cogenrerator in $A^{o p}$-mod. Moreover, if $\operatorname{gd}\left(\operatorname{End}_{A} V\right) \leq 3$, then $\operatorname{gd}\left(\operatorname{End}_{A^{o p}} \mathbf{D} V\right) \leq 3$, since $\operatorname{End}_{A^{o p}} \mathbf{D} V \simeq\left(\operatorname{End}_{A} V\right)^{o p}$. Finally, if $M \in \operatorname{add}_{A} V$, then $\mathbf{D} M \in \operatorname{add}_{A^{o p}} \mathbf{D} V$ and $\left(\operatorname{End}_{A} M\right)^{o p} \simeq \operatorname{End}_{A^{o p}} \mathbf{D} M$. Thus, the previous argument shows that $\operatorname{findim}\left(\operatorname{End}_{A} M\right)^{o p}\left(=\operatorname{findim}\left(\operatorname{End}_{A^{o p}} \mathbf{D} M\right)\right)$ is also finite.

Acknowledgement. Supported by the National Science Foundation of China (Grant Nos. 10971099 and 11171149) and by the Natural Science Foundation for Distinguished Young Scholars of Jiangsu Province (Grant No. BK2012044).

\section{REFERENCES}

1. D. Happel, Homological conjectures in representation theory of finite-dimensional algebras, Sherbrook Lecture Notes Series (Universite de Sherbrooke, 1991). Available at http://www.mathematik.uni-bielefeld.de/ sek/dim2/happel2.pdf, accessed 22 July 2013.

2. W. $\mathrm{Hu}$ and $\mathrm{C}$. Xi, Auslander-reiten sequences and global dimensions, Math. Res. Lett. 13(6) (2006), 885-895.

3. K. Igusa and G. Todorov, On the finitistic global dimension conjecture for artin algebras, in: Representations of algebras and related topics (Fields Inst. Commun. 45, American Mathematical Society, Providence, RI, 2005), 201-204.

4. F. Mantese and I. Reiten, Wakamatsu tilting modules, J. Algebra 278 (2004), 532-552.

5. C. M. Ringel, On the representation dimension of artin algebras, Bull. Inst Math. Acad. Sin. 7(1) (2012), 33-70.

6. A. Zhang and S. Zhang, On the finitistic dimension conjecture of artin algebras, J. Algebra 320(1) (2008), 253-258. 